\author{
Applied Mathematical Sciences, Vol. 12, 2018, no. 1, 19 - 26 \\ HIKARI Ltd, www.m-hikari.com \\ https://doi.org/10.12988/ams.2018.712351
}

\title{
The Expansion of the Confluent Hypergeometric Function on the Positive Real Axis
}

\author{
R. B. Paris \\ Division of Computing and Mathematics \\ Abertay University, Dundee DD1 1HG, UK
}

Copyright (C) 2018 R. B. Paris. This article is distributed under the Creative Commons Attribution License, which permits unrestricted use, distribution, and reproduction in any medium, provided the original work is properly cited.

\begin{abstract}
The asymptotic expansion of the Kummer function ${ }_{1} F_{1}(a ; b ; z)$ is examined as $z \rightarrow+\infty$ on the Stokes line arg $z=0$. The correct form of the subdominant algebraic contribution is obtained for non-integer a. Numerical results demonstrating the accuracy of the expansion are given.
\end{abstract}

Mathematics Subject Classification: 30E15, 33C15, 34E05, 41A60

Keywords: Confluent hypergeometric function, Stokes lines, asymptotic expansion

\section{Introduction}

The confluent hypergeometric function ${ }_{1} F_{1}(a ; b ; z)$ (or first Kummer function also denoted by $M(a, b, z))$ is defined for complex parameters $a$ and $b$ by

$$
{ }_{1} F_{1}(a ; b ; z)=\sum_{n=0}^{\infty} \frac{(a)_{n} z^{n}}{(b)_{n} n !}=1+\frac{a z}{b 1 !}+\frac{a(a+1) z^{2}}{b(b+1) 2 !}+\ldots,
$$

provided $b \neq 0,-1,-2, \ldots$, where $(a)_{n}=a(a+1) \ldots(a+n-1)=\Gamma(a+n) / \Gamma(a)$ is Pochhammer's symbol for the rising factorial. The series converges absolutely for all finite $z$ and reduces to a polynomial in $z$ when $a=0,-1,-2, \ldots$. 
We exclude this last case from our asymptotic considerations. The function ${ }_{1} F_{1}(a ; b ; z)$ is entire in $z$ and is consequently completely described in $-\pi<\arg z \leq \pi$.

The behaviour of ${ }_{1} F_{1}(a ; b ; z)$ for large $z$ and fixed parameters is exponentially large in $\Re(z)>0$ and algebraic in character in $\Re(z)<0$. The well-known asymptotic expansion of ${ }_{1} F_{1}(a ; b ; z)$ for $|z| \rightarrow \infty$ is given by $[3$, p. 328]

$$
\frac{\Gamma(a)}{\Gamma(b)}{ }_{1} F_{1}(a ; b ; z) \sim E(z)+H\left(z e^{\mp \pi i}\right)
$$

where the formal exponential and algebraic asymptotic series $E(z)$ and $H(z)$ are defined by

$$
E(z):=z^{a-b} e^{z} \sum_{j=0}^{\infty} \frac{(b-a)_{j}(1-a)_{j}}{j ! z^{j}}, \quad H(z):=\frac{z^{-a} \Gamma(a)}{\Gamma(b-a)} \sum_{k=0}^{\infty} \frac{(a)_{k}(1+a-b)_{k}}{k ! z^{k}} .
$$

In [3, p. 328], the sectors of validity of (1.1) are given by $-\frac{1}{2} \pi+\epsilon \leq \pm \arg z \leq$ $\frac{3}{2} \pi-\epsilon$, where $\epsilon>0$ denotes an arbitrarily small quantity. In the asymptotic theory of functions of hypergeometric type (see, for example, [6, §2], [7, §2.3]) the expansion (1.1) is given in the sector $-\pi<\arg z \leq \pi$, with the upper or lower sign being chosen according as $\arg z>0$ or $\arg z<0$, respectively. In the sense of Poincaré there is no inconsistency between these two sets of sectorial validity.

The exponential expansion $E(z)$ is dominant as $|z| \rightarrow \infty$ in $\Re(z)>0$ and becomes oscillatory on the anti-Stokes lines $\arg z= \pm \frac{1}{2} \pi$, where it is of comparable magnitude to the algebraic expansion. In $\Re(z)<0$, the exponential expansion is subdominant with the behaviour of ${ }_{1} F_{1}(a ; b ; z)$ then controlled by $H\left(z e^{\mp \pi i}\right)$. The negative real axis $\arg z= \pm \pi$ is a Stokes line where $E(z)$ is maximally subdominant. When $H\left(z e^{\mp \pi i}\right)$ is optimally truncated at, or near, its least term, the exponential expansion undergoes a smooth but rapid transition in the neighbourhood of arg $z= \pm \pi$; see [3, p. 67] and [7, Chapter 6]. It is clear, however, that (1.1) cannot account correctly for the exponentially small expansion on $\arg z=\pi$, since it predicts the exponentially small behaviour $\left(|z| e^{\pi i}\right)^{a-b} e^{-|z|}$ as $|z| \rightarrow \infty$. When $a-b$ is non-integer with $a$ and $b$ real, this is a complex-valued contribution whereas ${ }_{1} F_{1}(a ; b ;-|z|)$ is real.

The same argument applies on the other Stokes line arg $z=0$, where $E(z)$ is maximally dominant. The subdominant algebraic expansion undergoes a Stokes phenomenon as the positive real axis is crossed. According to the second set of validity conditions of (1.1), just above this ray the multiplicative factor in front of the algebraic expansion is $\left(z e^{-\pi i}\right)^{-a}$, whereas just below this ray the factor is $\left(z e^{\pi i}\right)^{-a}$. There will be a smooth transition (at fixed $|z|$ ) between these two expressions. The first set of validity conditions mentioned above is more confusing, since it appears that on $\arg z=0$ one has the choice 
of either $\left(z e^{-\pi i}\right)^{-a}$ or $\left(z e^{\pi i}\right)^{-a}$ for the multiplicative factors. In the case of real parameters, this predicts a complex-valued contribution from the subdominant expansion, when in fact it clearly must be real-valued.

Although such subdominant terms are negligible in the Poincaré sense, their inclusion can significantly improve the numerical accuracy in computations; see, for example, $\left[1\right.$, p. 76]. The details of the expansion of ${ }_{1} F_{1}(a ; b ; z)$ on the negative real axis have been discussed in [5]. In this note we complete this discussion by considering the correct form of the subdominant algebraic expansion as $z \rightarrow+\infty$ on the positive real axis.

\section{The expansion for ${ }_{1} F_{1}(a ; b ; x)$ as $x \rightarrow+\infty$}

In [5, Theorem 1], the expansion of the Kummer function ${ }_{1} F_{1}(a ; b ;-x)$ for $x \rightarrow+\infty$ was established, which took into account the correct asymptotic behaviour of the exponentially small contribution on the negative real axis. If we replace the parameter $a$ by $b-a$ in $[5,(2.11)]$, we have

$$
\begin{aligned}
& \frac{1}{\Gamma(b)}{ }_{1} F_{1}(b-a ; b ;-x)-\frac{x^{a-b}}{\Gamma(a)} \sum_{j=0}^{m_{0}-1} \frac{(b-a)_{j}(1-a)_{j}}{j ! x^{j}} \\
= & \frac{x^{-a} e^{-x}}{\Gamma(b-a)}\left\{\cos \pi a \sum_{j=0}^{M-1}(-)^{j} A_{j} x^{-j}+\frac{2 \sin \pi a}{\sqrt{2 \pi x}} \sum_{j=0}^{M-1}(-)^{j} B_{j} x^{-j}+O\left(x^{-M}\right)\right\},
\end{aligned}
$$

where the coefficients $A_{j}$ are given by

$$
A_{j}=\frac{(a)_{j}(1+a-b)_{j}}{j !} \quad(j \geq 0)
$$

and $M$ is a positive integer. Here, the dominant algebraic expansion on the left-hand side of (2.1), which has been optimally truncated with index $m_{o}$ given by

$$
m_{0}=x+\Re(2 a-b)+\alpha, \quad|\alpha|<1,
$$

has been subtracted off from ${ }_{1} F_{1}(b-a ; b ;-x)$. The expansion on the right-hand side represents the exponentially small contribution, where the coefficients $B_{j}$, which are related to the $A_{j}$, are specified below. If $a=n$, where $n$ is a positive integer, the sum on the left-hand side of (2.1) consists of $n$ terms and so cannot be optimally truncated. In this case, the expansion (2.1) holds with the upper limit of the sum on the left-hand side replaced by $n-1$ and with no contribution from the sum involving $B_{j}(\operatorname{since} \sin \pi a=0)$; see [5, Theorem 2].

The coefficients $B_{j}$ are defined by

$$
B_{j}=\sum_{k=0}^{j}(-2)^{k}\left(\frac{1}{2}\right)_{k} A_{j-k} G_{2 k, j-k} .
$$


The coefficients $G_{k, j}$ appear in the expansion of the so-called terminant function $T_{\nu}(z)$, which is defined as a multiple of the incomplete gamma function by $T_{\nu}(z):=\xi(\nu) \Gamma(1-\nu, z), \xi(\nu)=e^{\pi i \nu} \Gamma(\nu) /(2 \pi i)$. When

$$
\nu=m_{o}-2 a+b=x-\Im(2 a-b)+\alpha,
$$

so that $\nu \sim x$ as $x \rightarrow+\infty$, we have the expansion on the Stokes line arg $z=\pi$ of this function given by $[2, \S 5]$

$$
T_{\nu-j}\left(x e^{\pi i}\right)=\frac{1}{2}-\frac{i}{\sqrt{2 \pi x}}\left\{\sum_{k=0}^{M-1}\left(\frac{1}{2}\right)_{k} G_{2 k, j}\left(\frac{1}{2} x\right)^{-k}+O\left(x^{-M}\right)\right\},
$$

for $j=0,1,2, \ldots$ and positive integer $M$. The coefficients $G_{k, j}$ are computed from the expansion

$$
\frac{\tau^{\gamma_{j}-1}}{1-\tau} \frac{d \tau}{d w}=-\frac{1}{w}+\sum_{k=0}^{\infty} G_{k, j} w^{k}, \quad \frac{1}{2} w^{2}=\tau-\log \tau-1,
$$

where the parameter $\gamma_{j}$ is specified by

$$
\gamma_{j}=\nu-x-j=\alpha-j-\Im(2 a-b) \quad(0 \leq j \leq N-1)
$$

by (2.5). The branch of $w(\tau)$ is chosen such that $w \sim \tau-1$ as $\tau \rightarrow 1$ and so that upon reversion of the $w-\tau$ mapping

$$
\tau=1+w+\frac{1}{3} w^{2}+\frac{1}{36} w^{3}-\frac{1}{270} w^{4}+\frac{1}{4320} w^{5}+\cdots,
$$

it is found with the help of Mathematica that the first five even-order coefficients $G_{2 k, j} \equiv 6^{-2 k} \hat{G}_{2 k, j}$ are ${ }^{1}$

$$
\begin{aligned}
\hat{G}_{0, j}= & \frac{2}{3}-\gamma_{j}, \quad \hat{G}_{2, j}=\frac{1}{15}\left(46-225 \gamma_{j}+270 \gamma_{j}^{2}-90 \gamma_{j}^{3}\right), \\
\hat{G}_{4, j}= & \frac{1}{70}\left(230-3969 \gamma_{j}+11340 \gamma_{j}^{2}-11760 \gamma_{j}^{3}+5040 \gamma_{j}^{4}-756 \gamma_{j}^{5}\right), \\
\hat{G}_{6, j}= & \frac{1}{350}\left(-3626-17781 \gamma_{j}+183330 \gamma_{j}^{2}-397530 \gamma_{j}^{3}+370440 \gamma_{j}^{4}-170100 \gamma_{j}^{5}\right. \\
& \left.+37800 \gamma_{j}^{6}-3240 \gamma_{j}^{7}\right), \\
\hat{G}_{8, j}= & \frac{1}{231000}\left(-4032746+43924815 \gamma_{j}+88280280 \gamma_{j}^{2}-743046480 \gamma_{j}^{3}\right. \\
& +1353607200 \gamma_{j}^{4}-1160830440 \gamma_{j}^{5}+541870560 \gamma_{j}^{6}-141134400 \gamma_{j}^{7} \\
& \left.+19245600 \gamma_{j}^{8}-1069200 \gamma_{j}^{9}\right) .
\end{aligned}
$$

From this it is evident that the coefficients $B_{j}$ not only depend on $a$ and $b$ but also on $\alpha$ in (2.5), which in turn depends on the particular value of the variable $x$ under consideration.

\footnotetext{
${ }^{1}$ There was a misprint in the first term in $\hat{G}_{6, j}$ in [5], which appeared as -3226 instead of -3626. This was pointed out by T. Pudlik [4]. The correct value was used in the numerical calculations described in [5].
} 
If we apply Kummer's transformation

$$
{ }_{1} F_{1}(a ; b ; z)=e^{z}{ }_{1} F_{1}(b-a ; b ;-z)
$$

to the hypergeometric function in (2.1) we obtain

Theorem 1. When a is non-integer we have the expansion

$$
\begin{aligned}
& \frac{1}{\Gamma(b)}{ }_{1} F_{1}(a ; b ; x)-\frac{x^{a-b} e^{x}}{\Gamma(a)} \sum_{j=0}^{m_{o}-1} \frac{(b-a)_{j}(1-a)_{j}}{j ! x^{j}} \\
& =\frac{x^{-a}}{\Gamma(b-a)}\left\{\cos \pi a \sum_{j=0}^{M-1}(-)^{j} A_{j} x^{-j}+\frac{2 \sin \pi a}{\sqrt{2 \pi x}} \sum_{j=0}^{M-1}(-)^{j} B_{j} x^{-j}+O\left(x^{-M}\right)\right\}
\end{aligned}
$$

as $x \rightarrow+\infty$, where $m_{o}$ is the optimal truncation index defined in (2.3) satisfying $m_{o} \sim x$ and $M$ is a positive integer. The coefficients $A_{j}$ and $B_{j}$ are given in (2.2) and (2.4).

The expansion on the right-hand side of $(2.9)$ represents the correct form of the subdominant algebraic expansion of ${ }_{1} F_{1}(a ; b ; x)$ as $x \rightarrow+\infty$ on the Stokes line $\arg x=0$.

When $a=n$, where $n$ is a positive integer, the sum on the left-hand side of (2.9) terminates after $n$ terms (and so cannot be optimally truncated) and the sum on the right-hand side involving the coefficients $B_{j}$ vanishes. In this case, the expansion (2.9) reduces to the standard result in (1.1), where there is no ambiguity caused by the leading factor $\left(e^{\mp \pi i} x\right)^{-a}$ appearing in front of the algebraic expansion.

\section{Numerical examples and concluding remarks}

In this section we present some numerical examples to demonstrate the accuracy of the expansion in (2.9). As has already been noted, the coefficients $B_{j}$ depend on the parameters $a$ and $b$ and also on $\alpha$ (see the definition of $\gamma_{j}$ in (2.7)), which appears in the value of the optimal truncation index $m_{o}$ in (2.3). The value of $\alpha$ clearly is a function of the particular value of $x$ being considered. In Table 1 we show values of the coefficients $B_{j}$ for different $a, b$ and $\alpha$ (based on an integer value of $x$ ). In the calculations we have evaluated the coefficients $G_{2 k, j}$ for $0 \leq k \leq 6$.

In Table 2 we show the values of the quantity $\mathcal{F}(x)$ defined by the left-hand side of $(2.9)$

$$
\mathcal{F}(x):=\frac{1}{\Gamma(b)}{ }_{1} F_{1}(a ; b ; x)-\frac{x^{a-b} e^{x}}{\Gamma(a)} \sum_{j=0}^{m_{o}-1} \frac{(b-a)_{j}(1-a)_{j}}{j ! x^{j}}
$$


Table 1: Values of the coefficients $B_{j}$ (with $\gamma_{j}=\alpha-j-\Im(2 a-b)$ ) appearing in the expansion (2.9). The parameter $\alpha$ in (2.3) has been based on an integer value of $x$.

\begin{tabular}{c|c|c|c}
\hline$j$ & $\begin{array}{c}a=1 / 3, b=1 \\
\alpha=1 / 3\end{array}$ & $\begin{array}{c}a=3 / 4, b=1 / 2 \\
\alpha=0\end{array}$ & $\begin{array}{c}a=1 / 4, b=3 / 4 \\
\alpha=1 / 4\end{array}$ \\
\hline 0 & 0.33333333333 & 0.66666666667 & 0.41666666667 \\
1 & 0.15246913580 & 1.47731481481 & 0.16741898148 \\
2 & 0.15301905742 & 3.83399470899 & 0.18232822317 \\
3 & 0.35298391333 & 13.3434283401 & 0.44005533361 \\
4 & 1.17133694166 & 59.8043967080 & 1.48497131914 \\
5 & 4.97440127546 & 328.231235851 & 6.40730231996 \\
6 & 26.0263928534 & 2130.38975509 & 33.9936918351 \\
\hline
\end{tabular}

compared with the truncated right-hand side

$$
\mathcal{H}_{M}(x):=\frac{x^{-a}}{\Gamma(b-a)}\left\{\cos \pi a \sum_{j=0}^{M}(-)^{j} A_{j} x^{-j}+\frac{2 \sin \pi a}{\sqrt{2 \pi x}} \sum_{j=0}^{M}(-)^{j} B_{j} x^{-j}\right\}
$$

for different truncation index $M$. In each case the optimal truncation index $m_{o}$ of the exponential expansion in $\mathcal{F}(x)$ was determined by inspection and the value of $\alpha$ determined from (2.3). In the case with $a=\frac{1}{2}$ the sum involving the coefficients $A_{j}$ makes no contribution to $\mathcal{H}_{M}(x)$. It can be seen that the computed values of $\mathcal{F}(x)$ agree well with the subdominant algebraic expansion.

We remark that the so-called Stokes multiplier on the positive real axis (given by the quantity in curly braces on the left-hand side of (2.9)) is equal to $\cos \pi a$ to leading order. From (1.1) and (1.2) and the second set of validity conditions this quantity has the values $e^{\pi i a}$ and $e^{-\pi i a}$ just above and below $\arg z=0$. A commonly adopted, heuristic rule is that the Stokes multiplier on the Stokes line is given by the average of these two values to leading order, namely $\cos \pi a$. This agrees with the result stated in (2.9); see also [7, p. 248].

It would be of interest to extend the result of Theorem 1 to the more general Wright function defined by

$$
{ }_{1} \Psi_{1}\left(\begin{array}{c}
(\alpha, a) \\
(\beta, b)
\end{array} \mid z\right)=\sum_{n=0}^{\infty} \frac{\Gamma(\alpha n+a)}{\Gamma(\beta n+b)} \frac{z^{n}}{n !} \quad(\kappa>0,|z|<\infty),
$$

where $\alpha, \beta>0$ with $\kappa=1+\beta-\alpha$ and $a, b$ finite complex constants. From the asymptotic theory of functions of this type (see [6], $[7, \S \S 2.2 .4,2.3]$ ) we find that

$$
{ }_{1} \Psi_{1}\left(\begin{array}{c}
(\alpha, a) \\
(\beta, b)
\end{array} \mid z\right) \sim A Z^{\vartheta} e^{Z} \sum_{j=0}^{\infty} c_{j} Z^{-j}+\frac{1}{\alpha} \sum_{k=0}^{\infty} \frac{(-)^{k} \Gamma\left(\frac{k+a}{\alpha}\right)}{k ! \Gamma\left(b-\frac{\beta(k+a)}{\alpha}\right)}\left(z e^{\mp \pi i}\right)^{-(k+a) / \alpha}
$$


Table 2: Values of $\mathcal{F}(x)$ and $\mathcal{H}_{M}(x)$ using an optimal truncation $m_{o} \sim x$ of the exponential expansion for different index $M$ in the subdominant algebraic expansion and parameters $a$, $b$ when $x=20$.

\begin{tabular}{c||c|c|c} 
& $a=0.75, b=0.50$ & $a=0.50, b=1.25$ & $a=-0.75, b=1.25$ \\
$M$ & $\mathcal{H}_{M}(x)$ & $\begin{array}{c}a=-0.75, m_{o}=19 \\
\mathcal{H}_{M}(x)\end{array}$ & $\begin{array}{c}\alpha=0.75, m_{o}=18 \\
\mathcal{H}_{M}(x)\end{array}$ \\
\hline 0 & 0.01343919981 & 0.04612049350 & -6.58797674097 \\
1 & 0.01292521217 & 0.04684837963 & -6.29520877265 \\
2 & 0.01296951096 & 0.04689194291 & -6.29471792653 \\
3 & 0.01296356587 & 0.04689093247 & -6.29471024690 \\
4 & 0.01296463732 & 0.04689125453 & -6.29471013827 \\
5 & 0.01296439560 & 0.04689118984 & -6.29471013739 \\
6 & 0.01296446100 & 0.04689120714 & -6.29471013740 \\
\hline $\mathcal{F}(x)$ & 0.01296444571 & 0.04689120311 & -6.29471013740 \\
\hline
\end{tabular}

as $|z| \rightarrow \infty$ in the sector $|\arg z|<\min \{\pi, \pi \kappa\}$, with the upper or lower sign chosen according as $\arg z>0$ or $\arg z<0$, respectively. The various quantities in this asymptotic formula are given by

$$
Z=\kappa(h z)^{1 / \kappa}, \quad h=\alpha^{\alpha} \beta^{-\beta}, \quad \vartheta=a-b, \quad A=\kappa^{-\vartheta-\frac{1}{2}} \alpha^{a-\frac{1}{2}} \beta^{\frac{1}{2}-b}
$$

and the $c_{j}$ are coefficients with $c_{0}=1$ and

$$
\begin{gathered}
c_{1}=\frac{1}{12 \alpha \beta}\left\{\alpha(\alpha-1)\left(1-6 b+6 b^{2}\right)+\beta(\beta+1)\left(1-6 a+6 a^{2}\right)\right. \\
+\alpha \beta[\alpha-\beta-2(1+6 a b-6 b)]\} .
\end{gathered}
$$

Upon application of the above heuristic rule, we would expect the Stokes multiplier associated with the algebraic expansion on $\arg z=0$ to be equal to $\cos (\pi a / \alpha)$ to leading order. In the simpler case $\alpha=\beta$, a possible approach to investigate this conjecture is the integral representation

$$
{ }_{1} \Psi_{1}\left(\begin{array}{c}
(\alpha, a) \\
(\alpha, b)
\end{array} \mid z\right)=\frac{1}{\Gamma(b-a)} \int_{0}^{1} t^{a-1}(1-t)^{b-a-1} \exp \left[z t^{\alpha}\right] d t
$$

for $\Re(b)>\Re(a)>0$, which reduces to that of $(\Gamma(a) / \Gamma(b)){ }_{1} F_{1}(a ; b ; z)$ when $\alpha=1$. 


\section{References}

[1] F.W.J. Olver, Asymptotics and Special Functions, Academic Press, New York, 1974, Reprinted in A.K. Peters, Massachussets, 1997.

[2] F.W.J. Olver, Uniform, exponentially improved, asymptotic expansions for the generalized exponential integral, SIAM J. Math. Anal., 22 (1991), 1460-1474. https://doi.org/10.1137/0522094

[3] F.W.J. Olver, D.W. Lozier, R.F. Boisvert and C.W. Clark (eds.), NIST Handbook of Mathematical Functions, Cambridge University Press, Cambridge, 2010.

[4] T. Pudlik, Private communication, (2016).

[5] R.B. Paris, Exponentially small expansions of the confluent hypergeometric functions, Appl. Math. Sci., 7 (2013), 6601-6609.

https://doi.org/10.12988/ams.2013.310559

[6] R.B. Paris, The asymptotics of the generalised Bessel function, (2017), arXiv:1711.03006.

[7] R.B. Paris and D. Kaminski, Asymptotics and Mellin-Barnes Integrals, Cambridge University Press, Cambridge, 2001.

https://doi.org/10.1017/cbo9780511546662

Received: December 17, 2017; Published: January 5, 2018 